\title{
Comparison of the Efficiency of Piezoelectric Surgery, Er:Yag Laser and Rotary Surgical Burs in Harvesting Mandibular Block Grafts- an Ex-Vivo Study
}

\author{
J.Athiban Raj', Abhinav Rajendra Prabhu², Thiyaneswaran Nesappan³, Priya lochana \\ Gajendran ${ }^{2}$ and Subhasree. $R^{2}$ \\ ${ }^{1}$ Saveetha Dental College and Hospitals, Saveetha Institute of Medical and \\ Technical Sciences, Saveetha University, Chennai 600077, India \\ ${ }^{2}$ Senior Lecturer,Department of Implantology, Saveetha Dental College and Hospitals, Saveetha \\ Institute of Medical and Technical Sciences, Saveetha University, Chennai 600077, India \\ ${ }^{3}$ Professor \& Head of the Department,Department of Implantology, Saveetha Dental College and Hospitals, \\ Saveetha Institute of Medical and Technical Sciences, Saveetha University, Chennai 600077, India
}

\section{ABSTRACT}

Newer tools for bone surgery are available such as LASER's and piezoelectric units, which produce less thermal insult to bone and improved healing properties when compared to the conventional rotary burs. The aim of this study is to evaluate the most efficient method to harvest a mandibular block graft by using rotary bur, LASER and piezoelectric unit on cadaveric mandibles.This experimental ex-vivo study consisted of five cadaveric mandibles in which 15 sites were identified for block graft harvest.A block of size $5^{*} 5 \mathrm{~mm}$ was marked with an intended depth of osteotomy to be $3 \mathrm{~mm}$ and the osteotomy was performed.The harvested block graft and the resultant defect site were measured.Data was analysed using SPSS software.Mean value for volume of block graft was found to be comparatively less in rotary surgical bur group $(49.62 \pm 7.07 \mathrm{~mm} 3)$ and higher in the Piezoelectric group $(63.50 \pm 1.69 \mathrm{~mm} 3)(\mathrm{p}=0.005)$.Within the limitations of the current study, we conclude that piezo surgery is the best suited method to harvest a good viable bone graft when compared to LASER and Rotary burs.

KEY WORDS: AUTOGRAFT, BLOCK GRAFT, CADAVERIC MANDIBLE, PIEZOELECTRIC.

\section{ARTICLE INFORMATION}

*Corresponding Author: abhinav.sdc@saveetha.com

Received 13th June 2020 Accepted after revision 6th August 2020

Print ISSN: 0974-6455 Online ISSN: 2321-4007 CODEN: BBRCBA

Thomson Reuters ISI Web of Science Clarivate Analytics USA and Crossref Indexed Journal

$$
1 \text { Clarivate }{ }_{\text {Analytics }} \text { crossef }
$$

NAAS Journal Score 2020 (4.31) SJIF: 2020 (7.728)

A Society of Science and Nature Publication,

Bhopal India 2020. All rights reserved.

Online Contents Available at: http//www.bbrc.in/

Doi: $h$ ttp://dx.doi.org/10.21786/bbrc/13.7/37 


\section{INTRODUCTION}

Bone augmentation procedures are carried out in cases where there is a deficiency of the alveolar ridge, making it impossible to place implants for prosthetic restoration. Various techniques of bone augmentation have been employed to get the desired alveolar ridge dimensions. Various bone graft materials available are autografts, allografts, xenografts and alloplasts. The autografts are considered to be the gold standard in bone grafting materials as it has properties of osteoinduction as well as osteoconduction making it also highly osteogenic.

The commonest site of autograft harvest for block grafting is the mandibular symphysis region. The obvious reasons for an intra-oral site of graft harvest is the minimal scarring, absence of a skin incision, reduced operating time, procedure done on an outpatient basis (Nesappan and Ariga, 2014).The symphyseal region is considered a safe zone for surgical procedures and is easily accessible for graft harvest. Various methods are employed to harvest graft from this region. The earliest method used was using chisel and mallet, which has slowly lost its favour to more advanced methods using more modern equipment. Micromotors with rotary cutting burs, piezosurgery and use of LASER has been advocated in autograft harvest.(Gupta, Dhanraj and Sivagami, 2010) (Gupta, Dhanraj and Sivagami, 2010; Ashok et al., 2014) (Anbu et al., 2019)

Piezosurgery involves the use of piezoelectric burs, it is being used in Dental implant Surgery due to numerous advantages as they are precise and have selective cuttings, minimal or No thermal damage, and the preservation of soft-tissue structures.(Vidhya and Nesappan, 2016) It is not only used due to the advantage of very precise customized cutting but also due to factors associated with the healing process. The reduced blood loss improves healing conditions(Stübinger et al., 2008). Moreover, piezoelectric bone cutting does not influence bone remodeling or cell viability(Esteves et al., 2013).Rotary cutting burs are driven by micromotors which cause a mere mechanical ablation by crushing and shearing bone with serrated or diamond coated rotational hard metal bodies (Möhlhenrich et al., 2016). The action depends on size of the serrated surface, speed of the handpiece and pressure exerted onto the bone, thus causing frictional heat, which might cause bone necrosis. The major and unavoidable medical drawback of drills, burs and slowoscillating saws is the enormous procedural bone loss due to the minimum necessary diameter of the instrument of at least 1,5-2 $\mathrm{mm}$ and the imprecision of the cut due to the high torque-moment.(Rood, 1992)

Erbium-Yttrium-Aluminium-Garnet (Er:YAG) laser has emerged as a possible alternative to conventional methods of bone reduction. They have a high absorbability in water so it is highly safe to use it around implant during the treatment of bone reduction and peri-implantitis. (Romanos et al., 2009) (van As, 2004).It has many advantages such as noncontact, blood-reduced and vibration-reduced surgery techniques, free choice of cut geometry, a small operation field, and the prevention of massive bone flour and metal abrasion.(Pearson and Schuckert, 2003).

Previously our department has published extensive research on various aspects of dentistry including clinical trials (Anbu et al., 2019) (Venugopalan et al., 2014) (Abhinav et al., 2019) (Sweta, Abhinav and Ramesh, 2019) (Wahab et al., 2017) (Balaji and Gajendran, 2018) (Madhavan and Gajnedran, 2018) (Janani, Janani and Gajendran, 2018) ,in vitro studies (Ganapathy, Kannan and Venugopalan, 2017) (Ganapathy, Kannan and Venugopalan, 2017; Pandurangan, Veeraiyan and Nesappan, 2020) and systematic reviews (Kannan and Venugopalan, 2018). This vast experience has inspired us to research about the most efficient method to harvest symphysis block graft by comparing piezoelectric, LASER, Rotary cutting bur in cadaveric mandible.

\section{MATERIAL AND METHODS}

This was an experimental in-vitro study conducted at the Department of Implantology,Saveetha Dental College,Chennai ,India. Five cadaveric mandibles in which 15 sites were identified for block graft harvest were used for the study. The bone graft of size $5 \mathrm{~mm}$ length*5 mm width were marked on the mandibles using a graphite marker and markings were verified with Vernier caliper(Figure 1). The osteotomy was made using three cutting instruments- Rotary surgical burs(SS White Dental,USA), Piezoelectric unit (Piezotome Solo Acteon, France )and an Er:YAG LASER unit (Waterlase Iplus,USA) (Figure 2).

The osteotomy was done to a depth of $5 \mathrm{~mm}$ and was checked periodically to confirm the depth by using a graduated probe .Once the osteotomy was completed, the graft was taken with the use of a periosteal elevator (Figure 3). If the graft did not come out easily, a chisel and mallet was used to free the graft until the graft was free from the donor site.A stop watch was used to record the time taken from start of the osteotomy to the end of graft harvesting. After the graft harvest, the resultant defect was filled with gutta percha and a CBCT was taken for evaluation. The resultant defect site was measured using the CBCT software (GALAXIS-Galileos viewer 1.9) (Figure 4).All manual measurements were done using two methods-A graduated probe and by using a vernier caliper to prevent measurement bias.

\section{Volume calculation}

Volume of the graft was calculated using the following formula-

Volume of graft harvested $=$ Height of graft ${ }^{*}$ Width of the graft* Length of the graft

The loss of bone volume was calculated using the following formula:

Loss of bone volume $=$ Total volume of defect after graft harvest - Total volume of the harvested graft

Statistical Analysis: Data was analysed using SPSS software (IBM SPSS Statistics, Version 24.0, Amonk, 
NY: IBM Corp). Descriptive statistics were used for data summarization.Non-parametric Kruskal Wallis test was used to test for equality of the sample medians among the groups.p $<0.05$ was considered to be statistically significant.

\section{RESULTS AND DISCUSSION}

The mean values of Rotary surgical bur, Piezoelectric and Er.YAG LASER was calculated and tabulated (Table1). Mean value for volume of block graft was found to be comparatively less in Rotary surgical bur $(49.62 \pm 7.07$ mm3) and higher Piezoelectric $(63.50 \pm 1.69 \mathrm{~mm} 3)$.This was found to be statistically significant $(p=0.005)$. Mean value for volume of defect was found to be lowest in Er;YAG LASER (71.90 $\pm 6.61 \mathrm{~mm} 3)$ and highest in Rotary surgical bur (126.93 $\pm 6.58 \mathrm{~mm} 3)$.This was also statistically significant $(\mathrm{p}=.002)$. The mean value for time taken for graft harvest was lowest in rotary bur $(77.60 \pm 10.41$ secs $)$ and highest in Er;YAG LASER (503 \pm 49.17 secs). This was statistically significant( $p=0.002)$. Mean value for loss of bone volume compared to graft size was found to be lower in Er;YAG LASER (16.14 $\pm 6.40 \mathrm{~mm} 3)$ and higher in Rotary surgical bur $(77.30 \pm 8.30 \mathrm{~mm} 3)(\mathrm{p}=.002)$.

\begin{tabular}{l} 
Table 1. Outcome variables of the study \\
\hline
\end{tabular}

Mean value for volume of block graft was found to be comparatively less in Rotary surgical bur $(49.62 \pm 7.07$ $\mathrm{mm} 3)$ and higher Piezoelectric $(63.50 \pm 1.69 \mathrm{~mm} 3)$.This was found to be statistically significant( $p=0.005)$.It was maximum in piezoelectric unit as it was more precise and well controlled with proper irrigation whereas it is the least in rotary surgical bur as it has less control over the osteotomy. Based on the volume of defect, it is higher in Rotary surgical bur as it cuts excess bone due to its unpredicted control and stability whereas it is lesser in Er:YAG LASER.

Piezosurgery bone surgery has become famous off late due to its precise cutting, preservation of the soft tissues and lesser thermal heat when compared to rotary cutting drills (Birkholz, 1995).This results in enhanced operator sensitivity and control, indicating that the clinician can develop a far better 'feel' and precision for the cutting action thanks to micro vibration of cutting tip. The cutting action is smaller and less invasive, producing less collateral tissue damage, which ends up in better healing (Aro et al., 1981) (Crovace et al., 2020). The main disadvantage is its slowness. Cutting very dense bone with ultrasound can take up to 4 times longer than with a rotary bur. Tip breakage can be frequent which makes it necessary to maintain a stock of tips. The cost of ultrasonic osteotomy equipment is also elevated (González-García et al., 2009).

The use of piezosurgery in the field of implant dentistry is varied. It can be used to prepare implant osteotomies, harvest bone grafts, perform direct or indirect sinus lifts, perform ridge splits and very useful in nerve lateralizations.Otake et al. in their experimental study have shown that there is very minimal to no soft tissue injury when the piezoelectric saw is used on soft tissues. Similarly, when piezosurgery is performed on bones, it has been shown to have an increased viability of osteocytes and decreased cell death.A histomorphometric analysis study by Berengo et al. demonstrated that the amount of non vital bone was intermediate when compared with slow speed and high speed rotary burs (Berengo et al., 2006).Piezosurgery is also said to aid in faster healing of the surgical site decreased post-operative discomfort.

Figure 1: Graphite marking done on cadaveric mandible

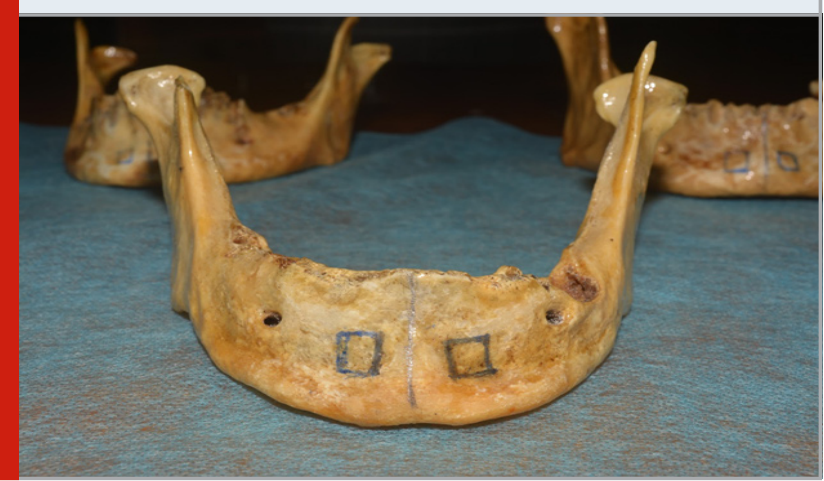

LASER surgery is fast becoming the new surgical aid for bone surgery due to its effects such as reduced pain and edema formation and rapid wound healing.The $2.94 \mu \mathrm{m}$ radiation emitted by the Er:YAG laser is ideal for absorption by water and hydroxyapatite, making it a very ideal candidate to perform hard and soft tissue LASER surgery (Hale and Querry, 1973) (Robertson and Williams, 1971). The advantages of cutting vital bone by Erbium lasers are a non-contact, reduced bleeding and non-vibrating form of surgery technique, free 
choice of cut geometry, a small operating field, and the prevention of massive bone necrosis and metal abrasion (Zolotarev et al., 1970).

However minimal knowledge, inadequate training, lesser experience of surgeons, and the overall cost often limit the use of LASER in everyday practice (Hale and Querry, 1973). Martins et al. compared bone healing between rotary burs and Er:YAG LASER osteotomies and concluded that bone healing was faster when surgical burs were used, with (Martins et al., 2011)similar results after 90 days of healing (Martins et al., 2011). We felt that though we had control over the LASER while performing the osteotomy, the perception of depth of the osteotomy was not possible. There was a need to stop and use an instrument to check for the depth throughout the procedure.

Figure 2: Osteotomy is done for Graft harvest using A) Piezoelectric B)LASER C)Rotary bur

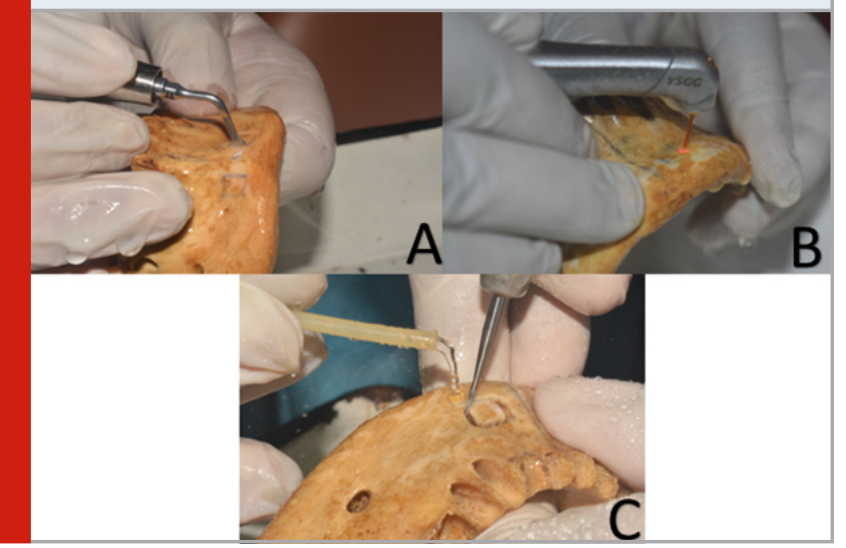

The rotary bur has been used far and wide and has a few advantages such as faster bone osteotomy, less pressure, greater ease of operation, economically feasible hardware to obtain. But its disadvantages are also a cause for concern.It produces excessive thermal damage thereby reducing the viability of the bone,increased vibrations and mechanical abrasions, soft tissue injury if not used cautiously and increased post-operative discomfort such as edema and pain.(Romeo et al., 2009). Schmidt in his study, showed that temperature rise was mainly related to formation of bone chips during cutting and their retention in the area thus clogging the bur. In our study our results showed that the highest volume of graft was harvested using a piezoelectric unit and highest volume of defect and mean bone loss was created while using a rotary bur to perform the graft harvest.

LASER surgery for graft harvest took a longer time to complete when compared to piezo surgery and use of rotary burs.From this we can conclude that piezo surgery is better compared to the other two modalities of bone surgery due to its least possibility of thermal necrosis, improved bone healing, high precision of the osteotomy design ,reduced mean bone loss, improved soft tissue preservation and reduced postoperative discomfort to the patient. The limitations of the study are that it is an ex- vivo study which cannot replicate the actual environment where these instruments would be potentially used in and the small sample size. Future studies should be aimed at designing long term clinical prospective studies which compare the intra-oral surgical time,post-operative complications and graft uptake in patients.

Figure 3: A) Block graft is elevated from the harvest site B) Defect region after removal of block graft

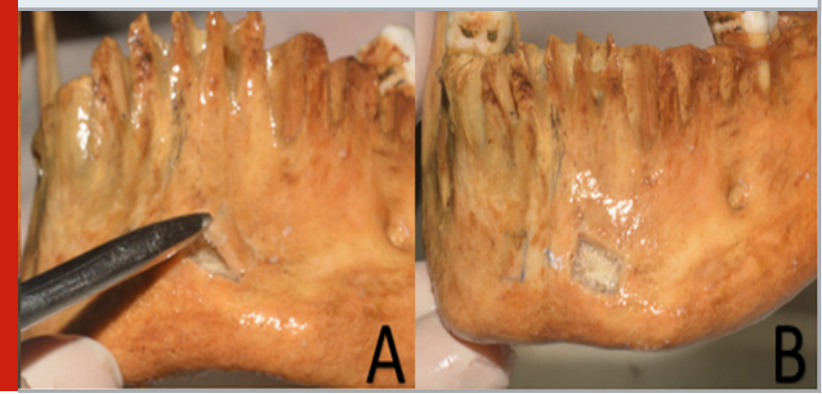

Figure 4: CBCT cross-section of the defect filled with gutta percha

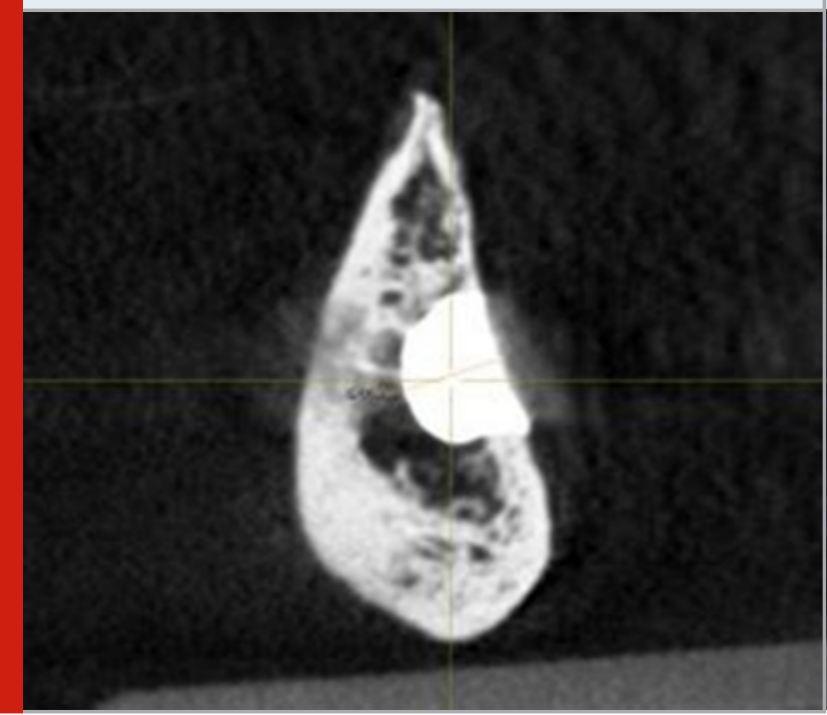

\section{CONCLUSION}

Within the limitations of the current study, we conclude that piezo surgery is the best suited method to harvest a good viable bone graft when compared to LASER and Rotary burs. Piezo surgery seems to define a possible new gold-standard in bone osteotomies due to their improved bone healing, reduced bone loss and precise osteotomy design and depth control, soft tissue protection as well as reduced intra surgical and post surgical morbidity

\section{Conflict of Interest: Nil}

\section{REFERENCES}

Abhinav, R. P. et al. (2019) 'The Patterns and Etiology of Maxillofacial Trauma in South India', Annals of maxillofacial surgery, 9(1), pp. 114-117. 
Anbu, R. T. et al. (2019) 'Comparison of the Efficacy of Three Different Bone Regeneration Materials: An Animal Study', European journal of dentistry, 13(1), pp. 22-28.

Aro, H. et al. (1981) 'Ultrasonic Device in Bone Cutting:A Histological and Scanning Electron Microscopical Study', Acta Orthopaedica Scandinavica, pp. 5-10. doi: 10.3109/17453678108991750.

van As, G. (2004) 'Erbium lasers in dentistry', Dental Clinics of North America, pp. 1017-1059. doi: 10.1016/j. cden.2004.06.001.

Ashok, V. et al. (2014) 'Lip Bumper Prosthesis for an Acromegaly Patient: A Clinical Report', Journal of Indian Prosthodontic Society, 14(Suppl 1), pp. 279282.

Balaji, S. and Gajendran, P. (2018) 'Correlation of Gingival Thickness with Gingival Width, Probing Depth and Papillary Fill in Mandibular Anterior Teeth', Research Journal of Pharmacy and Technology, p. 3918. doi: 10.5958/0974-360x.2018.00719.9.

Berengo, M. et al. (2006) 'Histomorphometric evaluation of bone grafts harvested by different methods', Minerva stomatologica, 55(4), pp. 189-198.

Birkholz, M. (1995) 'Crystal-field induced dipoles in heteropolar crystals II: Physical significance', Zeitschrift für Physik B Condensed Matter, pp. 333-340. doi: 10.1007/bf01313055.

Crovace, A. M. et al. (2020) 'Minimal Invasive Piezoelectric Osteotomy in Neurosurgery: Technic, Applications, and Clinical Outcomes of a Retrospective Case Series', Veterinary Sciences, p. 68. doi: 10.3390/ vetsci7020068.

Esteves, J. C. et al. (2013) 'Dynamics of bone healing after osteotomy with piezosurgery or conventional drilling - histomorphometrical, immunohistochemical, and molecular analysis', Journal of translational medicine, 11, p. 221.

Ganapathy, D. M., Kannan, A. and Venugopalan, S. (2017) 'Effect of Coated Surfaces influencing Screw Loosening in Implants: A Systematic Review and Metaanalysis', World Journal of Dentistry, pp. 496-502. doi: 10.5005/jp-journals-10015-1493.

González-García, A. et al. (2009) 'Ultrasonic osteotomy in oral surgery and implantology', Oral Surgery, Oral Medicine, Oral Pathology, Oral Radiology, and Endodontology, pp. 360-367. doi: 10.1016/j. tripleo.2009.04.018.

Gupta, A., Dhanraj, M. and Sivagami, G. (2010) 'Status of surface treatment in endosseous implant: a literary overview', Indian journal of dental research: official publication of Indian Society for Dental Research, 21(3), pp. 433-438.

Hale, G. M. and Querry, M. R. (1973) ‘Optical Constants of Water in the 200-nm to 200- $\mu \mathrm{m}$ Wavelength Region', Applied Optics, p. 555. doi: 10.1364/ao.12.000555.

Janani, Janani and Gajendran, P. L. (2018) 'Anti-Plaque Efficacy of cure next Gel, Hoira-sa gel in Comparison with Chlorhexidine Gel. A Randomised Control trial Study', Research Journal of Pharmacy and Technology, p. 3689. doi: 10.5958/0974-360x.2018.00677.7.

Kannan, A. and Venugopalan, S. (2018) 'A systematic review on the effect of use of impregnated retraction cords on gingiva', Research Journal of Pharmacy and Technology, p. 2121. doi: 10.5958/0974360x.2018.00393.1.

Madhavan, S. and Gajnedran, P. L. (2018) 'A Preliminary Study to compare The Pain Perception of Topical gel Versus Injected Local Infiltration/Block Anaesthesia during Non-Surgical Periodontal Therapy', Research Journal of Pharmacy and Technology, p. 4257. doi: 10.5958/0974-360x.2018.00780.1.

Martins, G. L. et al. (2011) 'Bone healing after bur and Er:YAG laser ostectomies', Journal of oral and maxillofacial surgery: official journal of the American Association of Oral and Maxillofacial Surgeons, 69(4), pp. 1214-1220.

Möhlhenrich, S. C. et al. (2016) 'Corrigendum to "Heat generation and drill wear during dental implant site preparation: systematic review" [Br. J. Oral Maxillofac. Surg. 53(8) (2015) 679-689]', The British journal of oral \&t maxillofacial surgery, 54(1), p. 117.

Nesappan, T. and Ariga, P. (2014) 'Comparison of Stresses Around Dental Implants Placed in Normal and Fibula Reconstructed Mandibular Models using Finite Element Analysis', Journal of clinical and diagnostic research: JCDR, 8(8), pp. ZC45-50.

Pandurangan, K., Veeraiyan, D. and Nesappan, T. (2020) 'In vitro evaluation of fracture resistance and cyclic fatigue resistance of computer-aided design-on and hand-layered zirconia crowns following cementation on epoxy dies', The Journal of Indian Prosthodontic Society, p. 90. doi: 10.4103/jips.jips_222_19.

Pearson, G. J. and Schuckert, K.-H. (2003) 'The Role of Lasers in Dentistry: Present and Future', Dental Update, pp. 70-76. doi: 10.12968/denu.2003.30.2.70.

Robertson, C. W. and Williams, D. (1971) 'Lambert Absorption Coefficients of Water in the Infrared*, Journal of the Optical Society of America, p. 1316. doi: 10.1364/josa.61.001316.

Romanos, G. E. et al. (2009) 'Laser wavelengths and oral implantology', Lasers in medical science, 24(6), pp. 961-970.

Romeo, U. et al. (2009) 'Bone damage induced by different cutting instruments: an in vitro study', Brazilian Dental Journal, pp. 162-168. doi: 10.1590/ s0103-64402009000200013. 
Rood, J. P. (1992) 'Permanent damage to inferior alveolar and lingual nerves during the removal of impacted mandibular third molars. Comparison of two methods of bone removal', British dental journal, 172(3), pp. 108-110.

Stübinger, S. et al. (2008) '[Ultrasonic bone cutting in oral surgery: a review of 60 cases]', Ultraschall in der Medizin , 29(1), pp. 66-71.

Sweta, V. R., Abhinav, R. P. and Ramesh, A. (2019) 'Role of Virtual Reality in Pain Perception of Patients Following the Administration of Local Anesthesia', Annals of maxillofacial surgery, 9(1), pp. 110-113.

Venugopalan, S. et al. (2014) 'Magnetically retained silicone facial prosthesis', Nigerian journal of clinical practice, 17(2), pp. 260-264.

Vidhya, G. and Nesappan, T. (2016) 'A piezoelectric surgery for direct sinus lift with immediate implant placement', Journal of Dental Implants, p. 79. doi: 10.4103/0974-6781.202158.

Wahab, P. U. A. et al. (2017) 'Risk Factors for Postoperative Infection Following Single Piece Osteotomy', Journal of Maxillofacial and Oral Surgery, pp. 328-332. doi: 10.1007/s12663-016-0983-6.

Zolotarev, V. M. et al. (1970) 'Dispersion and absorption of liquid water in infra-red and radio-frequency regions', Optics Communications, pp. 301-302. doi: 10.1016/0030-4018(70)90115-x. 